\title{
EDITORIALE
}

\section{Presentazione della rivista}

\author{
Luciano Marchi*
}

Perche una nuova rivista?

La rivista Management Control nasce per dare risposta alle richieste del mondo accademico e degli operatori aziendali rivolte, ci è sembrato di cogliere, ad un approfondimento delle tematiche di pianificazione, informazione e controllo, con il ricorso ad un approccio integrato di analisi.

Il nuovo approccio di analisi si riferisce alla necessaria integrazione dei sistemi operativi con quelli informativo-contabili, della struttura organizzativa con la tecnologia informatica, dell'analisi revisionale con quella prospettica, anche al fine di raccordare i sistemi di misurazione con quelli di governo aziendale, nel riferimento indistinto ad aziende pubbliche e private, profit e non-profit.

La rivista intende differenziarsi, in tal modo, dalle riviste che coprono le tematiche generali dell'economia aziendale e dall'insieme, più numeroso, delle riviste aventi carattere specialistico in rapporto ad una singola funzione aziendale ovvero ad uno specifico settore di attività.

\section{Con quale orientamento di fondo?}

La rivista si inserisce nel processo di internazionalizzazione della ricerca, con il fine di sviluppare gli studi teorici e applicativi, coniugando le conoscenze affermate a livello internazionale con i valori di fondo del pensiero economico aziendale italiano, anche nella scelta metodologica, cioè mantenendo il noto metodo di ricerca integrato induttivo-deduttivo.

\section{Con quali contenuti?}

Relativamente ai contenuti, la rivista intende trattare le tematiche dei sistemi di controllo aziendale, nella loro evoluzione come sistemi di controllo-guida, nei vari ambiti organizzativi ed ai vari livelli decisionali, dal livel-

\footnotetext{
*Università di Pisa; e-mail: lmarchi@ec.unipi.it
}

Management Control 1, 2011 
lo operativo a quello manageriale, dal livello strategico e quello "relazionale", con particolare riferimento alle seguenti aree tematiche:

1. sistemi di analisi delle prospettive, previsioni, business planning e controllo di gestione (area "Planning \& Budgeting");

2. sistemi di misurazione e di gestione dei costi e delle performance (area "Cost-Performance");

3. sistemi informativi e di reporting, nei vari aspetti di analisi, progettazione e gestione, incluse le componenti tecnologiche e quelle organizzative (area "Information Systems);

4. sistemi di audit gestionale, cioè di verifica dei caratteri di efficacia delle informazioni e delle operazioni di gestione, inoltre di analisi critica dei sistemi di controllo nel loro funzionamento effettivo (area "Management Audit").

Con quale controllo interno?

Gli studi e le ricerche proposti per la pubblicazione verranno sottoposti ad un doppio referaggio anonimo secondo gli standard internazionali. Preliminarmente verrà valutata la coerenza con la linea editoriale della rivista.

La qualità sarà inoltre garantita dall'alto profilo degli studiosi inseriti nel Comitato scientifico e nel Comitato editoriale, oltreché dal modello gestionale della rivista che prevede riunioni editoriali quadrimestrali di pianificazione degli obiettivi e controllo dei risultati e workshop scientifici semestrali sulle tematiche di Management Control.

Sempre al fine di garantire elevati standard scientifici, sono stati attivati processi di indicizzazione della rivista nell'ottica internazionale e di accreditamento AIDEA.

\section{Il primo numero della rivista}

Due parole chiave legano gli articoli contenuti nel presente numero di Management Control: innovazione e integrazione.

Con riferimento alla prima parola chiave, vengono formulate proposte sull'innovazione nei sistemi di controllo, sono considerati gli studi sviluppati in dottrina sulla nuova strumentazione di controllo, sono discussi i risultati di ricerche empiriche sull'utilizzo di sistemi e strumenti innovativi.

La trattazione si sviluppa a partire dai sistemi di costing con una ricerca comparativa sull'utilizzo dei nuovi modelli di Activity-Based Costing e Target Costing; ricerca svolta da Cinquini, Collini, Marelli e Tenucci che evidenzia due principali direzioni del cambiamento contabile: a) misurazioni che si ampliano, nella catena del valore, dalla vendita all'approvvigionamento; b) pro- 
cessi di misurazione che includono le dimensioni non lineari e asistematiche sul medio-lungo termine.

Gli articoli successivi prendono in considerazione lo sviluppo dei sistemi di misurazione delle performance nell'ottica "balanced scorecard" e dei sistemi informativi nell'ottica relazionale, per arrivare quindi all'innovazione dei sistemi di controllo interno nell'ottica della corporate governance e del risk management.

Con riferimento alla seconda parola chiave - l'integrazione nei sistemi di controllo - si possono osservare diversi livelli di integrazione, che forniscono anche una interpretazione della linea editoriale e delle scelte operate in termini di sequenza degli articoli proposti per il primo numero della rivista. Su questi diversi aspetti di integrazione si ritiene opportuno svolgere di seguito alcune considerazioni a carattere generale.

Il primo livello di integrazione e di orientamento gestionale, implicitamente considerato nella trattazione dei sistemi di rilevazione dei costi e delle performance, è quello che si realizza a consuntivo, nel passaggio dalle misurazioni di analisi alle determinazioni di sintesi, sia a livello fisicotecnico che a livello monetario.

Questo implica anche un approccio di audit informativo-contabile, con particolare riferimento alla verifica dei livelli di accuratezza ed ai caratteri di tempestività, rilevanza e selettività dei dati di sintesi/analisi.

Il secondo livello di integrazione, necessario ai fini del controllo risultati su obiettivi, è quello che si realizza tra i sistemi di consuntivazione e quelli di preventivazione, simulazione, pianificazione e controllo-guida nell'ottica prospettica. Questo passaggio è efficacemente considerato nel secondo articolo, a cura di Nicola Castellano, laddove si evidenzia che i comportamenti individuali e organizzativi sono largamente influenzati dalle misurazioni definite a preventivo e dagli indicatori di performance scelti per rappresentare gli obiettivi da raggiungere. L'integrazione determina, in questo caso, un forte bilanciamento tra misure interne ed esterne, inoltre tra misure finanziarie e non finanziarie, con una chiara esplicitazione dei nessi causali tra obiettivi strategici, obiettivi operativi, attività, risorse e competenze.

L'integrazione consuntivo-preventivo implica anche l'analisi critica dei dati, non solo mediante un audit sui caratteri di accuratezza, tempestività, rilevanza e selettività dei report di controllo, ma anche come analisi di coerenza interna dei dati a preventivo e di coerenza consuntivo su preventivo. A titolo esemplificativo, nell'analisi delle cause degli scostamenti risultati su obiettivi, uno scostamento rilevante determina tipicamente un feed-back sulla gestione (valutazione dell'efficacia interna) ovvero sulla pianificazione (revisione degli obiettivi, delle risorse e/o delle leve su cui agire per rag- 
giungere gli obiettivi). Con un approccio di audit potrebbe essere verificata anche l'accuratezza dei sistemi di misurazione dei risultati e la loro coerenza con i sistemi di misurazione degli obiettivi.

Sempre con riferimento a questo secondo livello di integrazione, vale la pena di precisare che il termine "controllo" può essere efficacemente utilizzato in senso lato, come controllo-guida, per esprimere sia le attività di analisi e valutazione dei risultati in rapporto agli obiettivi sia le attività di preventivazione, simulazione e pianificazione che, a monte, determinano le previsioni, le ipotesi e gli obiettivi stessi. Il termine controllo, così ampliato, include anche la guida, cioè il governo dei sistemi e l'orientamento delle azioni future.

Il terzo livello di integrazione è quello tra i dati nei loro diversi caratteri di provenienza e orientamento gestionale, anche in rapporto alla tecnologia utilizzata per realizzare la loro acquisizione ed il loro trattamento nell'ambito dei sistemi informatici, ai fini del controllo strategico: in particolare l'integrazione tra i dati interni e quelli esterni, tra i dati strutturati inseriti nel data-base aziendale ed i dati non strutturati gestiti in termini informali nelle relazioni interne ed esterne. Tra le innovazione più significative, con rilevante impatto sul controllo di gestione, rientra quella dei sistemi informativi relazionali trattata da Daniela Mancini nel terzo articolo, in termini generali, e da Marco Gatti nel quarto articolo, con specifico riferimento alle relazioni con i clienti.

L'evoluzione dei sistemi informativi nell'ottica strategica e relazionale presuppone, in ogni caso, significativi livelli di innovazione nell'utilizzo della tecnologia dell'informazione e della comunicazione (Information and Comunication Technology - ICT), dunque un efficace approccio di audit dei sistemi informatici, con particolare riferimento alla flessibilità e accettabilità dei sistemi ICT ed alla loro coerenza rispetto ai processi informativo-contabili, a monte, ed ai processi decisionali, a valle.

Il quarto livello di integrazione è quello relativo al necessario orientamento "comportamentale", nell'ambito della struttura organizzativa, che i dati e, più in generale, i sistemi di controllo interno devono realizzare nella loro struttura e nel loro funzionamento. Questo implica un approccio sia di audit gestionale che di controllo organizzativo.

Un approccio di audit gestionale comporta l'analisi critica delle operazioni/situazioni gestionali e delle correlate informazioni, interpretando la capacità dei sistemi manageriali di indirizzare i comportamenti individuali e collettivi verso gli obiettivi aziendali.

L'innovazione e l'integrazione che devono caratterizzare i sistemi informativi e, più in generale, i sistemi di controllo interno possono essere 
osservate da diversi punti di vista, ai vari livelli, nella prospettiva sia interna che esterna.

Il livello che qui interessa è ovviamente quello più elevato nella prospettiva interna, il livello del controllo di gestione, cioè del controllo-guida, che include la dimensione strategica e quella relazionale, e che deve orientare il management verso il conseguimento degli obiettivi di efficienza, efficacia ed economicità, sia particolare che generale.

Occorre rilevare, tuttavia, che per garantire un efficace controllo di gestione è necessario garantire l'efficacia dell'intero sistema di controllo interno, dunque anche il livello della compliance, in rapporto alla normativa ed alle direttive aziendali, ed il livello dell'affidabilità del sistema informativo contabile.

Inoltre, la prospettiva esterna, quella degli azionisti e, più in generale, dei diversi stakeholder presuppone che le caratteristiche dei sistemi di controllo interno siano comunicati all'esterno, affinché i vari stakeholder possano valutarne la qualità strutturale e l'efficacia di funzionamento.

Nell'articolo di Beretta, Bozzolan e Michelon si evidenzia, a tale riguardo, che il management aziendale ha maggiori incentivi alla disclosure quanto più deboli sono i meccanismi di governo aziendale.

Il problema di una limitata e non corretta informativa non si risolve imponendo una maggiore comunicazione ai mercati finanziari. L'aspetto rilevante dei sistemi di controllo interno è infatti quello della loro evoluzione nella direzione di una efficace governance, più nella prospettiva interna che nella prospettiva esterna. Su questa evoluzione, nelle aziende di maggiori dimensioni, è fondamentale il ruolo svolto dalla funzione Internal Auditing.

Il contributo che la suddetta funzione può fornire al miglioramento dei sistemi di controllo interno è trattato da D'Onza e Allegrini, a partire dai risultati di due ricerche empiriche condotte a livello internazionale, su tre direttrici di indagine: i processi di corporate governance, i processi di risk management ed i processi di responsabilità sociale.

La ricerca conferma le ipotesi discusse in precedenza sulla sempre maggiore proiezione esterna dei sistemi di controllo interno, a tutela degli interessi delle diverse classi di stakeholder. Si può affermare, a tale riguardo, che l'evoluzione del controllo interno nella prospettiva della corporate governance può tutelare gli interessi sia del management che degli investitori. La più recente evoluzione del controllo interno nella direzione del risk management, da un lato, e della responsabilità sociale, dall'altro, analogamente, può essere interpretata come la necessaria integrazione tra gli interessi interni e quelli esterni dei creditori, dei lavoratori e, più in generale, della comunità sociale. 
Più in generale, si ritiene che i sistemi di controllo interno debbano necessariamente integrare la dimensione economica e quella sociale, non solo nelle aziende pubbliche, ma anche nelle aziende private, a prescindere dalla loro finalità no-profit o profit. Ma alcune riflessioni finali si impongono sui rischi che allo sviluppo della comunicazione finanziaria e sociale non si accompagni un reale miglioramento della governance economico-sociale interna. Possibili risposte al problema sono le seguenti:

1) invertire la direzione dei processi di governance finanziaria: da un management che insegue gli interessi degli investitori-speculatori, specie quando entrano in gioco sistemi di stock options non correttamente impostati (teoria dell'agenzia) ad un management che indirizza le scelte degli investitori istituzionali, determinando relazioni cooperative;

2) analizzare, valutare e gestire i processi interni, passando da un approccio di risk management ad un approccio di management rischi-opportunità;

3) indirizzare in un'ottica interna e gestire in termini cooperativi le relazioni con i lavoratori e, più in generale, con la comunità sociale.

In conclusione, la prospettiva del controllo interno, inteso come controllo-guida, ai vari livelli di integrazione e innovazione, dovrebbe essere adeguatamente indagata, a livello teorico e applicativo. L'auspicio è che le riflessioni e le proposte fornite nel presente numero di Management Control possano stimolare il dibattito nei prossimi numeri della rivista. 